\title{
ON THE DISTRIBUTION OF RANK STATISTIC FOR STRONGLY CONCAVE COMPOSITIONS
}

\author{
NIAN HONG ZHOU
}

\begin{abstract}
A strongly concave composition of $n$ is an integer partition with strictly decreasing and increasing parts. In this paper we give a uniform asymptotic formula for the rank statistic of a strongly concave composition introduced by Andrews, Rhoades and Zwegers ['Modularity of the concave composition generating function', Algebra \& Number Theory 7 (2013), no. 9, 2103-2139].
\end{abstract}

\section{INTRODUCTION}

A partition of a positive integer $n$ is a sequence of non-increasing positive integers whose sum equals $n$. Let $p(n)$ be the number of integer partitions of $n$. To explain Ramanujan's famous partition congruences with modulus 5, 7 and 11, the rank and crank statistic for integer partitions was introduced by Dyson [8], Andrews and Garvan [2, 11]. Let $N(m, n)$ and $M(m, n)$ be the number of partitions of $n$ with rank $m$ and crank $m$, respectively. It is well known that

$$
\sum_{n \geq 0} N(m, n) q^{n}=\frac{1}{(q ; q)_{\infty}} \sum_{n \geq 1}(-1)^{n-1} q^{n(3 n-1) / 2+|m| n}\left(1-q^{n}\right),
$$

and

$$
\sum_{n \geq 0} M(m, n) q^{n}=\frac{1}{(q ; q)_{\infty}} \sum_{n \geq 1}(-1)^{n-1} q^{n(n-1) / 2+|m| n}\left(1-q^{n}\right),
$$

where $(a ; q)_{\infty}=\prod_{j \geq 0}\left(1-a q^{j}\right)$ for any $a \in \mathbb{C}$ and $|q|<1$.

In [9], Dyson gave the following asymptotic formulae conjecture for the crank statistic for integer partitions:

$$
M(m, n) \sim \frac{\pi}{4 \sqrt{6 n}} \operatorname{sech}^{2}\left(\frac{\pi m}{2 \sqrt{6 n}}\right) p(n), n \rightarrow+\infty .
$$

Bringmann and Dousse [4] proved (1.1) holds for all $|m| \leq(\sqrt{n} \log n) /(\pi \sqrt{6})$. Interestingly, Dousse and Mertens [10] proved (1.1) also holds for $N(m, n)$. For more results on asymptotics for rank and crank statistics for integer partitions, see $[6,7,13,14]$.

A concave composition $\lambda$ is a nonnegative integer sequence $\left\{a_{r}\right\}_{r=1}^{s}$ of the form

$$
a_{1} \geq a_{2} \geq \cdots \geq a_{k-1}>a_{k}<a_{k+1} \leq \cdots \leq a_{s-1} \leq a_{s},
$$

for some $s \in \mathbb{Z}_{+}$, where $a_{k}$ is called the central part of $\lambda$. If all the " $\geq$ " and " $\leq$ " are replaced by " $>$ " and " <", respectively, we refer to a strongly concave composition. The

2010 Mathematics Subject Classification. Primary: 11P82; Secondary: 05A16, 05A17.

Key words and phrases. concave composition, partitions, rank, asymptotics.

This research was supported by the National Science Foundation of China (Grant No. 11571114). 
rank of $\lambda$ above is defined as $\operatorname{rk}(\lambda):=s-2 k+1$, which analogs the rank statistic for integer partitions and measures the position of the central part.

Let $\mathcal{V}(n)$ and $\mathcal{V}_{d}(n)$ be the set of all concave composition and all strongly concave composition, respectively, of nonnegative integer $n$. Also, let $V(n)=\# \mathcal{V}(n)$ and $V_{d}(n)=\# \mathcal{V}_{d}(n)$ be the number of concave composition and strongly concave composition, respectively, of nonnegative integer $n$. Andrews [1] proved that

$$
v(q):=\sum_{n \geq 0} V(n) q^{n}=\sum_{n \geq 0} \frac{q^{n}}{\left(q^{n+1} ; q\right)_{\infty}^{2}}
$$

and

$$
v_{d}(q):=\sum_{n \geq 0} V_{d}(n) q^{n}=\sum_{n \geq 0}\left(-q^{n+1} ; q\right)_{\infty}^{2} q^{n}
$$

Andrews, Rhoades and Zwegers [3] proved that $v(q)$ is mixed modular form. More precise, they established the following modularity properties.

Theorem 1.1. Let $q=e^{2 \pi \mathrm{i} \tau}$ with $\tau \in \mathbb{C}$ and $\Im(\tau)>0$. Define $f(\tau)=q(q ; q)_{\infty}^{3} v(q)$ and

$$
\hat{f}(\tau)=f(\tau)-\frac{\mathrm{i}}{2} \eta(\tau)^{3} \int_{-\bar{\tau}}^{\mathrm{i} \infty} \frac{\eta(z)^{3}}{(-\mathrm{i}(z+\tau))^{1 / 2}} d z+\frac{\sqrt{3}}{2 \pi \mathrm{i}} \eta(\tau) \int_{-\bar{\tau}}^{\mathrm{i} \infty} \frac{\eta(z)}{(-\mathrm{i}(z+\tau))^{3 / 2}} d z
$$

where the Dedekind $\eta$-function is given by $\eta(\tau)=q^{1 / 24}(q ; q)_{\infty}$. The function $\hat{f}$ transforms as a modular form of weight 2 for $\mathrm{SL}_{2}(\mathbb{Z})$.

For $v_{d}(q)$, Andrews [1] proved that

$$
v_{d}(q)=2(-q ; q)_{\infty}^{2} \sum_{n \geq 0}\left(\frac{-12}{n}\right) q^{\frac{n^{2}-1}{24}}-\sum_{n \geq 0}(-1)^{n} q^{\frac{n(n+1)}{2}},
$$

where $(\dot{)})$ is the Kronecker symbol, that is, $v_{d}(q)+\sum_{n \geq 0}(-1)^{n} q^{\frac{n(n+1)}{2}}$ is essentially a modular function times a false theta function. And so we are not expect that the properties of $V_{d}(n)$ as good as $V(n)$. For example, [3] point out that it is possible to obtain an asymptotic with a polynomial error for $V(n)$ by use a circle method of Bringmann and Mahlburg [5], but for $V_{d}(n)$, we can’t seem to establish such asymptotic by existing approach. Nevertheless, [3, Theorem 1.5] gave the following asymptotic expansion ${ }^{\mathrm{a}}$

$$
V_{d}(N) \sim 2^{-1 / 4} 3^{-5 / 4} N^{-3 / 4} e^{2 \pi \sqrt{\frac{N}{6}}}\left(1+\sum_{n \geq 1} c_{n} N^{-n / 2}\right),
$$

for $N \rightarrow+\infty$, where $c_{n} \in \mathbb{R}, n \in \mathbb{Z}_{+}$is some computable constant.

\footnotetext{
at is need to note that the leading coefficient of the asymptotic expansion (1.2) is $2^{-1 / 4} 3^{-5 / 4}$ rather that $2 \cdot 2^{-1 / 4} 3^{-5 / 4}$ in [3, Theorem 1.5].
} 
Let $V_{d}(m, n)$ be the numbers of strongly concave compositions of $n$ with rank equal to $m$. Andrews, Rhoades and Zwegers [3] proved that ${ }^{\mathrm{b}}$

$$
\begin{aligned}
\sum_{n \geq 0} \sum_{m \in \mathbb{Z}} V_{d}(m, n) x^{m} q^{n}= & -\sum_{n \geq 0}(-1)^{n} q^{\frac{n(n+1)}{2}} x^{2 n+1} \\
& +(-x ; q)_{\infty}\left(-x^{-1} q ; q\right)_{\infty} \sum_{n \geq 0}\left(\frac{-12}{n}\right) x^{\frac{n-1}{2}} q^{\frac{n^{2}-1}{24}} .
\end{aligned}
$$

In this paper we investigate the asymptotics for $V_{d}(m, n)$ as $n$ tends to infinity with arbitrary $m$, which is motivated by the questions in [3, pp. 2108-2109] for the distribution of concave composition.

The first result of this paper as follows.

Proposition 1.2. Let $p(n)$ be the number of integer partitions of nonnative integer $n$ and let $p(-\ell)=0$ for $\ell \in \mathbb{Z}_{+}$. Then for $N, \ell \in \mathbb{Z}$ we have

$$
V_{d}\left(\ell, N+\frac{|\ell|(|\ell|+1)}{2}\right)=\sum_{n \geq 0}\left(\frac{-3}{2 n+1}\right) p\left(N-\frac{2 n(n+1)}{3}-n|\ell|\right) .
$$

In particular, for $m, n \in \mathbb{Z}$ with $0 \leq n<\frac{|m|(|m|+5)}{2}+4$,

$$
V_{d}(m, n)=p\left(n-\frac{|m|(|m|+1)}{2}\right) .
$$

From the above Proposition 1.2 we prove the following uniform asymptotics for $V_{d}(m, n)$ as $n \rightarrow+\infty$.

Theorem 1.3. We have uniformly for all $\ell \in \mathbb{Z}$ and $N \rightarrow+\infty$,

$$
V_{d}\left(\ell, N+\frac{|\ell|(|\ell|+1)}{2}\right)=p(N) F\left(\frac{\pi|\ell|}{\sqrt{6 N}}\right)\left(1+O\left(N^{-1 / 10}\right)\right),
$$

where the implied constant is absolute and

$$
F(\alpha)=\frac{1+e^{-\alpha}}{1+e^{-\alpha}+e^{-2 \alpha}} .
$$

In particular, if integer $m=o\left(N^{3 / 8}\right)$ then

$$
\frac{V_{d}(m, N)}{V_{d}(N)} \sim \frac{1}{(24 N)^{1 / 4}} \exp \left(-\frac{\pi m^{2}}{\sqrt{24 N}}\right) .
$$

We are going to statement the last one of results of this paper. Let the real function $\Psi_{d}(x)$ be defined as:

$$
\Psi_{d}(x)=\lim _{N \rightarrow+\infty} \frac{1}{V_{d}(N)} \#\left\{\lambda \in \mathcal{V}_{d}(N): \frac{\mathrm{rk}(\lambda)}{\left(6 N / \pi^{2}\right)^{1 / 4}} \leq x\right\}
$$

\footnotetext{
${ }^{\mathrm{b}}$ We correct some sign error in [3], where in $(1.3)$, it is $(-x ; q)_{\infty}\left(-x^{-1} q ; q\right)_{\infty} \sum_{n \geq 0}\left(\frac{-12}{n}\right) \ldots$ rather than $(x ; q)_{\infty}\left(x^{-1} q ; q\right)_{\infty} \sum_{n \geq 0}\left(\frac{12}{n}\right) \ldots$
} 
for $x \in \mathbb{R}$, which gives a limiting distribution of the rank statistic for strongly concave composition. It is clear that

$$
\begin{gathered}
\Psi_{d}(x)=\lim _{N \rightarrow+\infty} \frac{1}{V_{d}(N)} \sum_{\substack{m \in \mathbb{Z} \\
m \leq\left(6 N / \pi^{2}\right)^{1 / 4}}} \sum_{\substack{\lambda \in \mathcal{V}_{d}(N) \\
x \operatorname{rk}(\lambda)=m}} 1=\lim _{N \rightarrow+\infty} \sum_{\substack{m \in \mathbb{Z} \\
m \leq\left(6 N / \pi^{2}\right)^{1 / 4} x}} \frac{V_{d}(m, N)}{V_{d}(N)}, \\
\Psi_{d}(-\infty)=0 \text { and } \Psi_{d}(+\infty)=1 .
\end{gathered}
$$

Hence by using (1.7) and the fact that $V_{d}(m, N)=V_{d}(|m|, N)$, it is easy to deduce the following corollary by Abel's summation formula.

Corollary 1.4. We have for any fixed $x \in \mathbb{R}, \Psi_{d}(x)$ is the distribution function of the standard normal distribution, that is,

$$
\Psi_{d}(x)=\frac{1}{\sqrt{2 \pi}} \int_{-\infty}^{x} e^{-\frac{x^{2}}{2}} d x .
$$

Remark 1.1. The Corollary 1.4 is suggested by the referee.

\section{Proofs of Results}

\subsection{The proof of Proposition 1.2.}

By the Jacobi triple product formula

$$
(q ; q)_{\infty}(-x q ; q)_{\infty}\left(-x^{-1} ; q\right)_{\infty}=\sum_{n \in \mathbb{Z}} q^{\frac{n(n+1)}{2}} x^{n}
$$

and the basic properties of Kronecker symbol we obtain that

$$
\begin{aligned}
\sum_{\substack{n \geq 0 \\
m \in \mathbb{Z}}} V_{d}(m, n) x^{m} q^{n}= & -\sum_{n \geq 0}(-1)^{n} q^{\frac{n(n+1)}{2}} x^{2 n+1} \\
& +\frac{1}{(q ; q)_{\infty}} \sum_{\ell \in \mathbb{Z}} q^{\frac{\ell(\ell+1)}{2}} x^{-\ell} \sum_{n \geq 0}\left(\frac{-12}{2 n+1}\right) x^{n} q^{\frac{n(n+1)}{6}} .
\end{aligned}
$$

by using (1.3). This yields for integer $r \geq 0$,

$$
\begin{aligned}
\sum_{n \geq 0} V_{d}(-r, n) q^{n} & =\frac{1}{(q ; q)_{\infty}} \sum_{\substack{\ell-n=r \\
\ell \in \mathbb{Z}, n \geq 0}}\left(\frac{-3}{2 n+1}\right)\left(\frac{2}{2 n+1}\right)^{2} q^{\frac{n(n+1)}{6}+\frac{\ell(\ell+1)}{2}} \\
& =\sum_{k \geq 0} p(k) q^{k} \sum_{n \geq 0}\left(\frac{-3}{2 n+1}\right) q^{\frac{n(n+1)}{6}+\frac{(n+r)(n+r+1)}{2}} \\
& =\sum_{N \geq 0} q^{N} \sum_{n \geq 0}\left(\frac{-3}{2 n+1}\right) p\left(N-\frac{2 n(n+1)}{3}-r n-\frac{r(r+1)}{2}\right) .
\end{aligned}
$$

Which means that

$$
V_{d}\left(-\ell, N+\frac{\ell(\ell+1)}{2}\right)=\sum_{n \geq 0}\left(\frac{-3}{2 n+1}\right) p\left(N-\frac{2 n(n+1)}{3}-n \ell\right)
$$


for all integer $\ell \geq 0$. Thus by the fact that $V_{d}(-m, n)=V_{d}(m, n)$ we get the proof of (1.4) in Proposition 1.2. Further, if $2 \ell+4>N$ then

$$
V_{d}\left(\ell, N+\frac{|\ell|(|\ell|+1)}{2}\right)=p(N) .
$$

Namely we get the proof of (1.5) in Proposition 1.2.

2.2. Hardy-Ramanujan for $p(n)$ and its applications.

We need the following Hardy-Ramanujan asymptotic result for $p(n)$, which can be found in $[12]$.

Lemma 2.1. We have for $n \in \mathbb{Z}_{+}$,

$$
p(n)-\hat{p}(n-1 / 24)=O\left(n^{-1} e^{B \sqrt{n} / 2}\right),
$$

where $B=2 \pi / \sqrt{6}$ and

$$
\hat{p}(x)=\frac{e^{B \sqrt{x}}}{4 \sqrt{3} x}\left(1-\frac{1}{B \sqrt{x}}\right)
$$

(throughout, $B$ and $\hat{p}(x)$ be defined as above).

We also need the following approximation for $p(X+r)$ with $r=o\left(X^{3 / 4}\right)$.

Lemma 2.2. Let $r=o\left(X^{3 / 4}\right)$ and $X$ be sufficiently large, we have

$$
\frac{p(X+r)}{p(X)}=e^{\frac{B r}{2 \sqrt{X}}}\left(1+O\left(\frac{1}{X}+\frac{|r|}{X}+\frac{|r|^{2}}{X^{3 / 2}}\right)\right) .
$$

Proof. From Lemma 2.1, it is clear that

$$
\begin{aligned}
\frac{\hat{p}(X+r)}{\hat{p}(X)} & =e^{B(\sqrt{X+r}-\sqrt{X})}\left(1+O\left(\frac{|r|}{X}\right)\right) \\
& =e^{\frac{B r}{2 \sqrt{X}}+O\left(r^{2} / X^{3 / 2}\right)}\left(1+O\left(\frac{|r|}{X}\right)\right) \\
& =e^{\frac{B r}{2 \sqrt{X}}}\left(1+O\left(\frac{|r|}{X}+\frac{|r|^{2}}{X^{3 / 2}}\right)\right)
\end{aligned}
$$

by the generalized binomial theorem. Note that

$$
\frac{p(N)}{\hat{p}(N)}=1+O\left(\frac{1}{N}\right)
$$

holds for all $N \geq 1$ we obtain that

$$
\frac{p(X+r)}{p(X)}=e^{\frac{B r}{2 \sqrt{X}}}\left(1+O\left(\frac{1}{X}+\frac{|r|}{X}+\frac{|r|^{2}}{X^{3 / 2}}\right)\right) .
$$

Which completes the proof of the lemma.

\subsection{The proof of Theorem $\mathbf{1 . 3}$.}


2.3.1. Case $|\ell|>\sqrt{N}(\log N)^{2}$. We first denote

$$
F(\ell, N):=V_{d}\left(\ell, N+\frac{|\ell|(|\ell|+1)}{2}\right) .
$$

For $N / 2 \geq|\ell|>\sqrt{N}(\log N)^{2}$, from Proposition 1.2 and Lemma 2.2 we obtain that

$$
\begin{aligned}
F(\ell, N) & =\sum_{\substack{n \geq 0 \\
2 n(n+1) / 3+n|\ell| \leq N}}\left(\frac{-3}{2 n+1}\right) p\left(N-\frac{2 n(n+1)}{3}-n|\ell|\right) \\
& =p(N)+O\left(\sum_{\substack{n \geq 2 \\
2 n(n+1) / 3+n \ell \leq N}} p(N-n|\ell|)\right) \\
& =p(N)+O(\sqrt{N} p(N-2|\ell|))=p(N)+O\left(\sqrt{N} p\left(N-\left\lfloor\sqrt{N}(\log N)^{2}\right\rfloor\right)\right) \\
& =p(N)\left(1+O\left(\sqrt{N} \exp \left(-\frac{B\left\lfloor\sqrt{N}(\log N)^{2}\right\rfloor}{2 \sqrt{N}}\right)\right)\right),
\end{aligned}
$$

where $\lfloor\cdot\rfloor$ is the greatest integer function. Hence, we have if $N / 2 \geq|\ell|>\sqrt{N}(\log N)^{2}$ then

$$
F(\ell, N)=p(N)\left(1+O\left(N^{-\sqrt{\log N}}\right)\right) .
$$

2.3.2. Case $|\ell| \leq \sqrt{N}(\log N)^{2}$. If $0 \leq \ell \leq \sqrt{N}(\log N)^{2}$ since

$$
\left(\frac{-3}{2 n+1}\right)=\left\{\begin{array}{cl}
1 & \text { if } n \equiv 0 \bmod 3 \\
0 & \text { if } n \equiv 1 \bmod 3 \\
-1 & \text { if } n \equiv 2 \bmod 3
\end{array}\right.
$$

we have

$$
\begin{aligned}
F(\ell, N) & =\sum_{n \geq 0}\left(\frac{-3}{2 n+1}\right) p\left(N-\frac{2 n(n+1)}{3}-n \ell\right) \\
& =\sum_{n \geq 0}\left[p\left(N-Q_{1}(n, \ell)\right)-p\left(N-Q_{2}(n, \ell)\right)\right],
\end{aligned}
$$

where

$$
Q_{1}(n, \ell)=2 n(3 n+1)+3 n \ell \text { and } Q_{2}(n, \ell)=Q_{1}(n, \ell)+(8 n+4+2 \ell) .
$$

We split that

$$
\begin{aligned}
\frac{F(\ell, N)}{p(N)}= & \frac{1}{p(N)} \sum_{\substack{n \geq 0 \\
n^{2}+n \ell>\sqrt{N}(\log N)^{2}}}\left[p\left(N-Q_{1}(n, \ell)\right)-p\left(N-Q_{2}(n, \ell)\right)\right] \\
& +\frac{1}{p(N)} \sum_{\substack{n \geq 0 \\
n^{2}+n \ell \leq \sqrt{N}(\log N)^{2}}}\left[p\left(N-Q_{1}(n, \ell)\right)-p\left(N-Q_{2}(n, \ell)\right)\right]=: R+I .
\end{aligned}
$$


Noting that $Q_{2}(n, \ell) \geq Q_{1}(n, \ell) \geq n^{2}+n \ell$ for all $n \geq 0$ we estimate that

$$
\begin{aligned}
|R| & \leq \frac{2}{p(N)} \sum_{\substack{n \geq 0 \\
n^{2}+n \ell>\sqrt{N}(\log N)^{2}}} p\left(N-Q_{1}(n, \ell)\right) \\
& \leq \frac{2}{p(N)} \sum_{\substack{n \geq 0 \\
n^{2}+n \ell>\sqrt{N}(\log N)^{2}}} p\left(N-\left(n^{2}+n \ell\right)\right) \leq 2 \sqrt{N} \frac{p\left(N-\left\lfloor\sqrt{N}(\log N)^{2}\right\rfloor\right)}{p(N)} .
\end{aligned}
$$

Thus Lemma 2.2 implies the following estimate for $R$,

$$
R \ll \sqrt{N} e^{-\frac{B\left\lfloor\sqrt{N}(\log N)^{2}\right\rfloor}{2 \sqrt{N}}} \ll N^{-\sqrt{\log N}} .
$$

To estimate $I$, we note that

$$
0 \leq Q_{1}(n, \ell) \leq Q_{2}(n, \ell) \leq 16\left(n^{2}+n \ell\right)+2 \ell+4=O\left(\sqrt{N}(\log N)^{2}\right),
$$

if $n \geq 0$ and $n^{2}+n \ell \leq \sqrt{N}(\log N)^{2}$. Then by Lemma 2.2 we have

$$
\begin{aligned}
I= & \sum_{n \geq 0}\left(e^{-\frac{B Q_{1}(n, \ell)}{2 \sqrt{N}}}-e^{-\frac{B Q_{2}(n, \ell)}{2 \sqrt{N}}}\right)-\sum_{\substack{n \geq 0 \\
n^{2}+n \ell>\sqrt{N}(\log N)^{2}}}\left(e^{-\frac{B Q_{1}(n, \ell)}{2 \sqrt{N}}}-e^{-\frac{B Q_{2}(n, \ell)}{2 \sqrt{N}}}\right) \\
& +O\left(\sum_{i=1}^{2} \sum_{\substack{n \geq 0 \\
n^{2}+n \ell \leq \sqrt{N}(\log N)^{2}}} e^{-\frac{B Q_{i}(n, \ell)}{2 \sqrt{N}}}\left(\frac{1}{N}+\frac{Q_{i}(n, \ell)}{N}+\frac{Q_{i}(n, \ell)^{2}}{N^{3 / 2}}\right)\right)=I_{M}+I_{R}
\end{aligned}
$$

with

$$
I_{M}=\sum_{n \geq 0}\left(e^{-\frac{B Q_{1}(n, \ell)}{2 \sqrt{N}}}-e^{-\frac{B Q_{2}(n, \ell)}{2 \sqrt{N}}}\right)
$$

and

$$
\begin{aligned}
I_{R} & \ll \sum_{\substack{n \geq 0 \\
n^{2}+n \ell>\sqrt{N}(\log N)^{2}}} e^{-\frac{B\left(n^{2}+\ell n\right)}{\sqrt{N}}}+\sum_{\substack{n \geq 0 \\
n^{2}+n \ell \leq \sqrt{N}(\log N)^{2}}} \frac{(\log N)^{4}}{N^{1 / 2}} e^{-\frac{B\left(n^{2}+n \ell\right)}{2 \sqrt{N}}} \\
\ll N^{-\sqrt{\log N}}+N^{-1 / 2}(\log N)^{4} & \sum_{\substack{n \geq 0 \\
n^{2}+n \ell \leq \sqrt{N}(\log N)^{2}}} 1 \ll N^{-1 / 4}(\log N)^{5} .
\end{aligned}
$$

From the above, we conclude above that

$$
F(\ell, N) / p(N)=I_{M}+O\left(N^{-1 / 5}\right)
$$

holds for $0 \leq \ell \leq \sqrt{N}(\log N)^{2}$.

For the estimate of $I_{M}$ we need the following lemma.

Lemma 2.3. Let $0 \leq \ell=o\left(\alpha^{-1}\right)$, then as $\alpha \rightarrow 0^{+}$,

$$
f(\alpha):=\alpha \sum_{n \geq 0}(4 n+\ell) e^{-2 \alpha n^{2}-\alpha n \ell}=1+O(\sqrt{\alpha}+|\alpha \ell|) .
$$


Proof. By Abel's summation formula or integration by parts for a Riemann-Stieltjes integral we obtain that

$$
\begin{aligned}
f(\alpha) & =4 \alpha \sum_{n \geq 0}(n+\ell / 4) e^{-2 \alpha(n+\ell / 4)^{2}+\frac{\alpha \ell^{2}}{8}} \\
& =4 \alpha e^{\frac{\alpha \ell^{2}}{8}} \int_{0-}^{\infty} e^{-2 \alpha(x+\ell / 4)^{2}} d\left(\sum_{0 \leq n \leq x}(n+\ell / 4)\right) \\
& =4 \alpha e^{\frac{\alpha \ell^{2}}{8}}\left(\int_{0}^{\infty} e^{-2 \alpha(x+\ell / 4)^{2}} d\left(\frac{x^{2}}{2}+\frac{x \ell}{4}\right)+O\left(\alpha \int_{0}^{\infty}(x+\ell / 4)^{2} e^{-2 \alpha(x+\ell / 4)^{2}} d x\right)\right) \\
& =4 \alpha e^{\frac{\alpha \ell^{2}}{8}}\left(\int_{\ell / 4}^{\infty} x e^{-2 \alpha x^{2}} d x+O\left(\alpha \int_{\ell / 4}^{\infty} x^{2} e^{-2 \alpha x^{2}} d x\right)\right) \\
& =e^{\frac{\alpha \ell^{2}}{8}} \int_{\alpha \ell^{2} / 8}^{\infty} e^{-x} d x+O\left(\sqrt{\alpha} e^{\frac{\alpha \ell^{2}}{8}} \int_{\alpha \ell^{2} / 8}^{\infty} x^{1 / 2} e^{-x} d x\right)=1+O(\sqrt{\alpha}+|\alpha \ell|),
\end{aligned}
$$

which completes the proof of the lemma.

We now evaluate $I_{M}$. By the definition of $F(\alpha)$ and $I_{M}$, it is clear that if $\ell \geq N^{3 / 8}$ then

$$
\begin{aligned}
I_{M} & =\sum_{0 \leq n \leq N^{1 / 5}}\left(e^{-\frac{B Q_{1}(n, \ell)}{2 \sqrt{N}}}-e^{-\frac{B Q_{2}(n, \ell)}{2 \sqrt{N}}}\right)+O\left(N^{-\sqrt{\log N}}\right) \\
& =\sum_{0 \leq n \leq N^{1 / 5}} e^{-\frac{B(3 n+1) n}{\sqrt{N}}}\left(1-e^{-\frac{B(\ell+4 n)}{\sqrt{N}}}\right) e^{-\frac{3 B n \ell}{2 \sqrt{N}}}+O\left(N^{-\sqrt{\log N}}\right) \\
& =\left(1+O\left(N^{-1 / 10}\right)\right) \sum_{0 \leq n \leq N^{1 / 5}}\left(1-e^{-\frac{B(\ell+4 n)}{\sqrt{N}}}\right) e^{-\frac{3 B n \ell}{2 \sqrt{N}}}+O\left(N^{-\sqrt{\log N}}\right) \\
& =\left(1+O\left(N^{-1 / 10}\right)\right) \frac{1-e^{-\frac{B \ell}{\sqrt{N}}}}{1-e^{-\frac{3 B \ell}{2 \sqrt{N}}}}=\left(1+O\left(N^{-1 / 10}\right)\right) F\left(\frac{B \ell}{2 \sqrt{N}}\right)
\end{aligned}
$$

and if $0 \leq \ell \leq N^{3 / 8}$ then

$$
\begin{aligned}
I_{M} & =\sum_{0 \leq n \leq N^{2 / 5}}\left(e^{-\frac{B n}{\sqrt{N}}}-e^{-\frac{B(5 n+\ell)}{\sqrt{N}}}\right) e^{-\frac{B\left(6 n^{2}+3 n \ell\right)}{2 \sqrt{N}}}+O\left(N^{-\sqrt{\log N}}\right) \\
& =\left(1+O\left(N^{-1 / 10}\right)\right) \sum_{0 \leq n \leq N^{2 / 5}} \frac{B(4 n+\ell)}{\sqrt{N}} e^{-\frac{B\left(6 n^{2}+3 n \ell\right)}{2 \sqrt{N}}}+O\left(N^{-\sqrt{\log N}}\right) \\
& =\left(1+O\left(N^{-1 / 10}\right)\right) \frac{B}{\sqrt{N}} \sum_{n \geq 0}(4 n+\ell) e^{-\frac{B\left(6 n^{2}+3 n \ell\right)}{2 \sqrt{N}}}+O\left(N^{-\sqrt{\log N}}\right) \\
& =\frac{2}{3}\left(1+O\left(N^{-1 / 10}\right)\right)\left(1+O\left(N^{-1 / 4}+\ell N^{-1 / 2}\right)\right)=\left(1+O\left(N^{-1 / 10}\right)\right) F\left(\frac{B \ell}{2 \sqrt{N}}\right)
\end{aligned}
$$

by the use of Lemma 2.3. Thus it is clear that for $0 \leq \ell \leq \sqrt{N}(\log N)^{2}$,

$$
F(\ell, N)=p(N) F\left(\frac{\pi \ell}{\sqrt{6 N}}\right)\left(1+O\left(N^{-1 / 10}\right)\right)
$$

by use (2.3) and $B=2 \pi / \sqrt{6}$. 
Finally, by using (2.1), (2.2), (2.4) and the fact that $V_{d}(m, n)=V_{d}(|m|, n)$ we finish the proof of (1.6). By using (1.2), (1.6) and Lemma 2.1 we obtain the proof of (1.7), which completes the proof of Theorem 1.3.

\section{ACKNOWLEDGMENT}

The author would like to thank the referee for very helpful and detailed comments and suggestions.

\section{REFERENCES}

[1] G. E. Andrews. Concave and convex compositions. Ramanujan J., 31(1-2):67-82, 2013.

[2] G. E. Andrews and F. G. Garvan. Dyson's crank of a partition. Bull. Amer. Math. Soc. (N.S.), 18(2):167$171,1988$.

[3] G. E. Andrews, R. C. Rhoades and S. P. Zwegers. Modularity of the concave composition generating function. Algebra Number Theory, 7(9):2103-2139, 2013.

[4] K. Bringmann and J. Dousse. On Dyson's crank conjecture and the uniform asymptotic behavior of certain inverse theta functions. Trans. Amer. Math. Soc., 368(5):3141-3155, 2016.

[5] K. Bringmann and K. Mahlburg. An extension of the Hardy-Ramanujan circle method and applications to partitions without sequences. Amer. J. Math., 133(4):1151-1178, 2011.

[6] K. Bringmann and J. Manschot. Asymptotic formulas for coefficients of inverse theta functions. Commun. Number Theory Phys., 7(3):497-513, 2013.

[7] K. Byungchan, K. Eunmi and S. Jeehyeon. Asymptotics for $q$-expansions involving partial theta functions. Discrete Math., 338(2):180-189, 2015.

[8] F. J. Dyson. Some guesses in the theory of partitions. Eureka, (8):10-15, 1944.

[9] F. J. Dyson. Mappings and symmetries of partitions. J. Combin. Theory Ser. A, 51(2):169-180, 1989.

[10] J. Dousse and M. H. Mertens. Asymptotic formulae for partition ranks. Acta Arith., 168(1):83-100, 2015.

[11] F. G. Garvan. New combinatorial interpretations of Ramanujan's partition congruences mod 5, 7 and 11. Trans. Amer. Math. Soc., 305(1):47-77, 1988.

[12] G. H. Hardy and S. Ramanujan. Asymptotic Formulae in Combinatory Analysis. Proc. London Math. Soc. (2), 17:75-115, 1918.

[13] R. Mao. Asymptotic inequalities for $k$-ranks and their cumulation functions. J. Math. Anal. Appl., 409(2):729-741, 2014.

[14] D. Parry and R. C. Rhoades. On Dyson's crank distribution conjecture and its generalizations. Proc. Amer. Math. Soc., 145(1):101-108, 2017.

School of Mathematical Sciences, East China Normal University, 500 Dongchuan Road, Shanghai 200241, PR China

E-mail address: nianhongzhou@outlook.com 